\title{
Guest Editorial Foreword to the Special Issue of IHC 2018
}

This special issue of JIS presents the extended versions of the best full papers of the Brazilian Symposium on Human Factors in Computing Systems (IHC 2018) on its twentieth anniversary. In these 20 years, IHC has consolidated itself as the Brazilian HCI community's main event, and has played a relevant role in the establishment of the HCI community itself. IHC 2018 was held in Belém, from October 22 to 26. In 2018, there were 166 valid submissions for full papers, and 42 were accepted (25.3\% acceptance rate). This special issue presents the seven papers selected as best papers from the 42 presented at the conference. It is worth noting that their extended versions present original content or new contributions when compared to their original IHC 2018 version, and they were submitted to a new and independent review process.

IHC 2018 highlighted the influence and importance of cultural issues in the design of computer systems, as well as the need to be creative and innovative in designing new forms of interaction, design, and system evaluations with its theme "Interaction, Culture, and Creativity". The seven papers selected for this issue tap into IHC 2018's theme and represent the broad range of topics being investigated in HCI in Brazil, as well as bring original and relevant contributions to the stateof-the-art in each of their topics.

In the first paper in this issue, Chagas, Redmiles and de Souza focus on the appropriation of emerging technology, namely IoT technology used in smart homes. As a result, they present a semiotic account of appropriation and propose two derived qualities that can be relevant in addressing appropriation of IoT technologies: cross-interface consistency (consistency between different interfaces of a single device) and crossdevice coherence (defines that when devices are combined they should all together form a minimally unified and harmonic whole). They point out that these qualities call for new evaluation methods and design practices for IoT.

Whereas Chagas and co-authors bring to light issues related to IoT, an emerging technology, in the next paper, Duarte, Merkle and Baranauskas explore an issue that can be considered of interest for centuries: the articulation between art and science. More specifically the authors explore the articulation between interactive art and HCI scientific contributions. To do so, they investigate what kind of humancomputer interactions emerge in interactive arts, and also what kinds of frameworks, evaluation criteria and methodologies support the design and evaluation of interactive arts. Their results show that interactive arts have mainly focused on four dialogue genres (or interactive paradigms): virtual, embodied, tangible and social interaction. Their research indicates that evaluation methods, especially those focused on user experience, can be a common ground between the two fields. They conclude that the articulation of art and science can be an important source of innovation for interactive systems and $\mathrm{HCI}$, and propose a research agenda in this direction.

These first two papers, discuss the need for new evaluation methods that could tackle specific aspects they each have investigated and discussed. In this direction, the following paper presents a novel set of inspection techniques for a different, but well-known issue: assessing privacy in online social networks (OSN). Rodrigues, Valentim and Feitosa propose and describe the Privacy Inspection Techniques for Online Social Network (PIT-OSN). They also present a preliminary study performed to evaluate their proposal. Results showed that PIT-OSN helped non-experts uncover privacy problems, and indicate that its application can be useful in the development, evaluation and research of privacy in OSNs.

The next paper, also brings to attention a socially relevant issue, albeit of a very different nature. Within the domain of post-mortem technology, Pereira et al. explore young adults' perspective of Digital Legacy Management Systems (DLMS). They investigated through two case studies whether young adults were able to understand the new concepts introduced by the DLMS domain, and their experience in interacting with such systems. Their results indicated that participants understood the new concepts well, but had difficulties in grasping how processes that would only take place in the future would work. As for their experience, they associated the use of the system in any role (account owner, trustee or heir) as having a high emotional cost. These results could indicate challenges for DLMS adoption.

The following paper, brings to light a very current and important issue, as Paim and Prietch focus on the challenge for deaf and hard of hearing people in learning a written language. In order to support the development of assistive technology aimed at this challenge, they propose a co-design framework comprised of by four phases: Explore and Understand; Identify Needs, Design and Develop; Evaluate and Monitor. In this paper, they illustrate the application of phase 1 (Explore and Understand) through a case-study. Their results show that phase 1 of the framework allowed stakeholders to bring forth central issues to them, such as cultural diversity and multimodality, that were then considered in the design of the 
assistive technology designed aimed at deaf and sign language users.

Cascaes et al. also take into consideration special needs, but from a different perspective. In their paper, the authors focus on supporting the identification of children that may have special needs. Deviations from typical child development, for instance dyslexia or autism spectrum disorder, can be uncovered by assessing the child's strategies for visual exploration. Cancellation tests comprise a subgroup of visual exploration tests that can be used for that purpose. In this paper, the authors propose an automated version of a cancellation test and perform two evaluation tests with children. Their results indicate that the automated test can collect more data and guarantee greater accuracy, besides allowing the applicator to focus on the participants' other behavioral aspects not collected by the test. Furthermore, the automated tool allows for the creation of databases of test execution that can be useful in future studies of visual exploration in association with cognitive functions.

Finally, the last paper of our issue, as the first, is interested in appropriation, but in a different context. Müller, Silveira and de Souza investigate how novice programmers learn existing source code, and how they appropriate this code and reuse it in their own code. In order to do so, they take a semiotic perspective and examine what novices communicate through their source code. As a result, they identified three different reuse and appropriation scenarios: (1) authorial: in which participants wrote their code from scratch; (2) non-authorial: participants reused an existing example, but did not completely understand how it worked; and (3) co-authorial: participants reused an example and grasped how it worked. Based on these scenarios they discuss different levels of understanding and appropriation that can be useful not only for researchers, but also for educators or even professionals interested in analyzing or assessing programmers' reuse of code.

This overview shows that the papers in this special edition cover a broad spectrum of current HCI issues. Each of them present a detailed account of their research questions and findings, which we hope will not only be interesting and useful to readers, but also spark their scientific curiosity and foster their following-up with the authors' continuing researches, or tackling the future works proposed themselves.

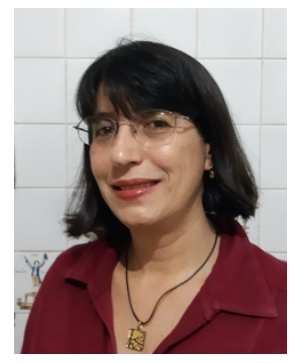

Raquel O. Prates is an Associate Professor of the Computer Science Department at Federal University of Minas Gerais where she coordinates PENSi (Research Group in Semiotic Engineering and Interaction). She received her PhD from the Department of Informatics at PUC-Rio in 1998 and is an active member of the HCI and Collaborative Systems communities, nationally and internationally, having participated in the Executive Committee for HCI (SIGCHI and CEIHC), and Collaborative Systems (CESC), as well as in Program and Technical Committees for conferences and acted as a reviewer for a number of journals. E-mail: rprates@dcc.ufmg.br

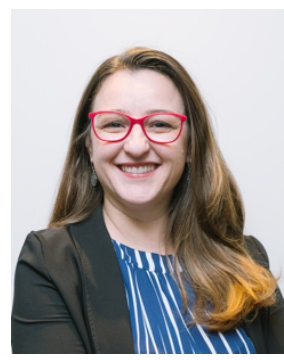

Heloisa Candello is a researcher and an interaction designer at the Conversational Intelligence group at IBM Research laboratory in Brazil. She holds a PhD. in Computer Science, Interactive Technologies from the University of Brighton - UK. Before joining IBM, Heloisa was a lecturer and a researcher in mobile interaction technologies. Nowadays, at IBM, Heloisa led and designed novel and extraordinary user experiences and research in the context of conversational systems. Her research resulted in several publications in leading conferences (CHI, CSCW, DRS, DUXU, IUI) and recognition in the HCI and Design field. Heloisa was also an organizer of several workshops and cochair of conferences in the field. Currently, Heloisa is a full paper co-chair of CUI2020.

E-mail: heloisacandello@br.ibm.com 